\title{
Neuere Anschauungen über die Konstitution und Reaktionsweise der Borwasserstoffverbindungen
}

\author{
Von F. SEEL \\ Aus dem Anorgan.-chem. Institut der Technischen Hochschule München
}

(Z. Naturforschg. 1, 146-150 [1946]; eingegangen am 20. November 1945)

\begin{abstract}
Es wird das vielerörterte Valenzproblem der Borwasserstoffverbindungen erneut aufgegriffen und versucht, zu einer befriedigenden Lösung zu gelangen. Zunächst kann nachgewiesen werden, daß sich die meisten chemischen Reaktionen des Diborans $\mathrm{B}_{2} \mathrm{H}_{6}$ durch die Wirksamkeit der Komponente $\mathrm{BH}_{3}$, ,Borin“ erklären lassen. Als Ursache der Dimerisierung des Borins wird die Wechselwirkung zweier BH-Bindungen erkannt. Schließlich wird die Zusammensetzung, Molekulargröße und Struktur der höhermolekularen Borhydride theoretisch einwandfrei abgeleitet und gezeigt, daß keine weitere flüchtige Verbindung zu erwarten ist. - Infolge der Begrenztheit des zur Verfügung stehenden Raumes wurde auf eine Diskussion der bestehenden älteren Theorien über die Konstitution der Borane verzichtet. Es sei an dieser Stelle nur bemerkt, daß die Annahme einer Analogie zwischen den Strukturen des Diborans $\mathrm{B}_{2} \mathrm{H}_{6}$ einerseits und Äthans $\mathrm{C}_{2} \mathrm{H}_{6}$ oder Äthylens $\mathrm{C}_{2} \mathrm{H}_{4}$ andererseits unbegründet ist und zu keiner richtigen Lösung des Problems führt.

Die nachfolgende Abhandlung stellt die stark gekürzte Wiedergabe einer ausführlichen Arbeit dar, welche bereits am 10. Oktober 1943 zur Veröffentlichung in der Zeitschrift für physikalische Chemie eingereicht wurde, aber infolge widriger Umstände vor Kriegsende nicht mehr erscheinen konnte.
\end{abstract}

$\mathrm{D}$ ie Konstitutionsfrage der Borwasserstoffverbindungen ist eines der interessantesten Probleme der anorganischen Chemie. Den Grund hierfür erkennt man sofort bei der Betrachtung der Summenformeln der bis jetzt bekannten flüchtigen Hydride: $\mathrm{B}_{2} \mathrm{H}_{6}, \mathrm{~B}_{4} \mathrm{H}_{10}, \mathrm{~B}_{5} \mathrm{H}_{11}, \mathrm{~B}_{5} \mathrm{H}_{9}, \mathrm{~B}_{6} \mathrm{H}_{12}, \mathrm{~B}_{6} \mathrm{H}_{10}$, $\mathrm{B}_{10} \mathrm{H}_{14}$. Deren Zusammensetzung erscheint ja recht eigenartig, besonders wenn man hiermit die leicht zu deutenden Formeln der Kohlenwasserstoffe vergleicht z. B. $\mathrm{CH}_{4}, \mathrm{C}_{2} \mathrm{H}_{6}, \mathrm{C}_{3} \mathrm{H}_{8}$ usw., allgemein $\mathrm{C}_{\mathrm{n}} \mathrm{H}_{2 \mathrm{n}+2}$. Schon das Anfangsglied in der Reihe der Borane entspricht nicht der Formel $\mathrm{BH}_{3}$, welche man auf Grund der Existenz monomerer Halogenide und Alkyle $\mathrm{BX}_{3}$ hierfür erwarten sollte. Es hat vielmehr die doppelte Molekulargröße $\left(\mathrm{BH}_{3}\right)_{2}$ oder $\mathrm{B}_{2} \mathrm{H}_{6}$.

Das chemische Verhalten des Diborans $\mathrm{B}_{2} \mathrm{H}_{6}$ kann jedoch in seinen wesentlichsten Zügen bereits durch die einfache Formulierung $\mathrm{BH}_{3}$ wiedergegeben werden, wodurch sich der einfachste Borwasserstoff dennoch stetig in die Reihe der übrigen Verbindungen des dreiwertigen Bors eingliedert. Es ist anzunehmen, daß die hier anzuführenden Reaktionen des Diborans auf eine Dissoziation fol-

1 H. I. Schlesinger, N. W. Flodin u. A. B. Burg, J. Amer. chem. Soc. 61, 1078 [1939]. gen, welche erst während der Umsetzung stattzufinden braucht $\left(\mathrm{BH}_{3}\right)_{2}+\mathrm{A} \rightarrow \mathrm{A} \cdot \mathrm{BH}_{3}+\mathrm{BH}_{3}$. Die Frage nach der Ursache der Polymerisation der Komponente $\mathrm{BH}_{3}$, „Borin“, ist hierbei zunächst natürlich gegenstandslos.

Die Úberlegung, daß Methyldiboran $\mathrm{BH}_{3}$ . $\mathrm{BH}_{2}\left(\mathrm{CH}_{3}\right)$ unter bestimmten Bedingungen so reagiert, als ob es in die Komponenten Borin und Methylborin zerfallen sei, ermöglichte die Darstellung des symm. Dimethyldiborans durch Abfangen des Borins mit Dimethyläther ${ }^{1}$ :

$$
\begin{aligned}
\mathrm{BH}_{3} \cdot \mathrm{BH}_{2}\left(\mathrm{CH}_{3}\right) & \longrightarrow \mathrm{BH}_{3}+\mathrm{BH}_{2}\left(\mathrm{CH}_{3}\right) \\
\mathrm{BH}_{3}+\mathrm{O}\left(\mathrm{CH}_{3}\right)_{2} & \longrightarrow \mathrm{BH}_{3} \mathrm{O}\left(\mathrm{CH}_{3}\right)_{2} \\
2 \mathrm{BH}_{2}\left(\mathrm{CH}_{3}\right) & \longrightarrow \mathrm{BH}_{2}\left(\mathrm{CH}_{3}\right)^{2} \cdot \mathrm{BH}_{2}\left(\mathrm{CH}_{3}\right)
\end{aligned}
$$

Bei vielen Reaktionen tritt das Diboran nur ,monomer" in Wirkung, indem das Radikal Borin im Molekül des Endproduktes nur einfach auftritt. So vereinigt es sich mit Trimethylamin $\mathrm{zu} \mathrm{BH}_{3} \mathrm{~N}\left(\mathrm{CH}_{3}\right)_{3}$ und mit Kohlenoxyd zu $\mathrm{BH}_{3} \mathrm{CO}$ („Borincarbonyl ${ }^{{ }^{* 2} 2}$ ). Auch die Alkalimetall-Addition ${ }^{3}$, in welcher man eine Parallele zu dem Verhalten der ungesättigten Kohlenwasserstoffe gefunden zu haben

2 A. B. B u r g u. H. I. S chl e singer, J. Amer. chem. Soc. 59, 780 [1937].

3 A. Stock, F. Kurzen u. H. Laudenklos, Z. anorg. allg. Chem. 225, 225 [1935].
Dieses Werk wurde im Jahr 2013 vom Verlag Zeitschrift für Naturforschung in Zusammenarbeit mit der Max-Planck-Gesellschaft zur Förderung der Wissenschaften e.V. digitalisiert und unter folgender Lizenz veröffentlicht: Creative Commons Namensnennung-Keine Bearbeitung 3.0 Deutschland Lizenz.

Zum 01.01.2015 ist eine Anpassung der Lizenzbedingungen (Entfall de Creative Commons Lizenzbedingung „Keine Bearbeitung“) beabsichtigt, um eine Nachnutzung auch im Rahmen zukünftiger wissenschaftlicher Nutzungsformen zu ermöglichen.
This work has been digitalized and published in 2013 by Verlag Zeitschrift für Naturforschung in cooperation with the Max Planck Society for the Advancement of Science under a Creative Commons Attribution-NoDerivs 3.0 Germany License. 
glaubte, läßt sich durch die Wirksamkeit des koordinativ ungesättigten Borins erklären: Entsprechend der Bildung des Triphenylborkaliums ${ }^{4}$ aus Bortriphenyl entsteht primär ein Salz mit einem Radikal als Anion, welches sofort dimerisiert ${ }^{5}$.

$$
\begin{gathered}
\mathrm{B}\left(\mathrm{C}_{6} \mathrm{H}_{5}\right)_{3}+\mathrm{K} \longrightarrow\left[. \mathrm{B}\left(\mathrm{C}_{6} \mathrm{H}_{5}\right)_{3}\right] \mathrm{K} \\
\mathrm{BH}+\mathrm{K} \longrightarrow\left[. \mathrm{BH}_{3} \mid \mathrm{K}\right. \\
2\left[. \mathrm{BH}_{3}\right] \mathrm{K} \longrightarrow\left[\mathrm{B}_{2} \mathrm{H}_{6}\right] \mathrm{K}_{2}
\end{gathered}
$$

Die zahlreichen, charakteristischen und bei den Kohlenwasserstoffen völlig fehlenden anionischen Substitutionsreaktionen des Diborans haben ihre Ursache darin, daß der Wasserstoff den elektronegativen Molekülbestandteil bildet. Wasserstoff steht nämlich in der Skala der relativen Elektronenaffinitäten (scale of electronegativity) ${ }^{\circ}$ zwischen Bor und Kohlenstoff, wodurch die Ladungsinkremente der $\mathrm{B}-\mathrm{H}-\mathrm{Bindung}$ gerade in dem entgegengesetzten Sinne verteilt sind wie bei der C-H-Bindung:

$$
\begin{array}{ll}
\delta+\delta- & \delta-\delta+ \\
\mathrm{B}-\mathrm{H} & \mathrm{C}-\mathrm{H} .
\end{array}
$$

Deshalb führt ebenso wie die Hydrolyse der Borwasserstoffbindung

$$
\begin{array}{ll}
\delta+\delta-\delta+\delta- & \delta+\delta- \\
\mathrm{B}-\mathrm{H}+\mathrm{H}-\mathrm{OH} & \mathrm{B}-\mathrm{OH}+\mathrm{H}_{2}
\end{array}
$$

auch die Einwirkung anderer protonenaktiver Stoffe, wie von Alkoholen und Halogenwasserstoffen, zu Wasserstoffentbindung, wobei dessen Stelle leicht durch negative Gruppen ersetzt wird und die Substitution auch auf verschiedenen Stufen stehen bleiben kann.

$$
\begin{aligned}
\mathrm{BH}_{3}+2 \mathrm{CH}_{3} \mathrm{OH} & \longrightarrow \mathrm{BH}\left(\mathrm{OCH}_{3}\right)_{2}+\mathrm{H}_{2}{ }^{7} \\
\left(\mathrm{BH}_{3}\right)_{2}+\mathrm{HJ} & \longrightarrow \mathrm{BH}_{3} \mathrm{BH}_{2} \mathrm{~J}^{+}+\mathrm{H}_{2} \mathrm{~s}^{2}
\end{aligned}
$$

Die negative Natur des Borin-Wasserstoffes tritt fernerhin in der Bildung anionischer Wasserstoffkomplexe wie $\left[\mathrm{BH}_{4}\right] \mathrm{Li}^{9},\left(\mathrm{BH}_{4}\right)_{2} \mathrm{Be}^{10}$ zutage, sowie in seiner Fähigkeit als Anion zu wandern (Anionotropie) : Die Einwirkung von Carbonylverbin-

4 E. Krause, Ber. dtsch. chem. Ges. 57, 216 [1924].

5 Vergl. L. K l e m m u. W. K l e m m, Z. anorg. allg. Chem. 225, 258 [1935].

6 Vergl. L. Pa uling, J. Amer. chem. Soc. 54, 3570 [1932]; R. S. M ulliken, J. chem. Physics 73, 1 [1931].

7 A. B. B urg u. H. I. Schlesinger, J. Amer. chem. Soc. 55, 4020 [1933].

8 A.Stock u. E. Pohland, Ber. dtsch. chem. Ges. 59, 2223 [1926].

${ }^{9}$ H. I. Schlesinger u. H. C. Brown, J. Amer. chem. Soc. 62, 3429 [1940].

10 A. B. B u r g u. H. I. S chlesinger, J. Amer. chem. Soc. 62, 3425 [1940]. dungen auf Diboran führt über die nach dem Verhalten des Borfluorides ${ }^{11}$ zu erwartende Molekülverbindung $\mathrm{R}_{2} \mathrm{CO} . \mathrm{BH}_{3}$ hinausgehend zu Alkoxyboranen ${ }^{12}$ :<smiles></smiles>

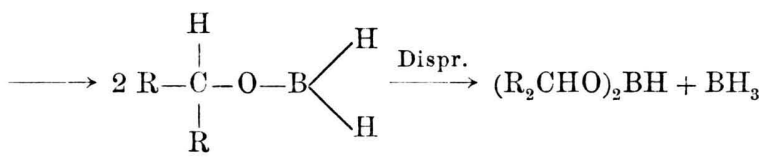

Endlich läßt sich die Hypoboratbildung des Diborans, $\left(\mathrm{BH}_{3}\right)_{2}+2 \mathrm{KOH} \rightarrow\left[\mathrm{BH}_{2} \mathrm{OH} . \mathrm{BH}_{2} \mathrm{OH}\right] \mathrm{K}_{2}$ $+\mathrm{H}_{2}$ über ein primäres Hydroxoanion des Borins $\left[\mathrm{B}_{\mathrm{OH}}^{\mathrm{H}_{3}}\right]$ gehend formulieren, welches anschließend unter Wasserstoffabgabe dimerisiert:

$$
\begin{aligned}
\mathrm{BH}_{3}+\mathrm{OH}^{-} & \longrightarrow\left[\mathrm{B}_{\mathrm{OH}}^{\mathrm{H}_{3}}\right] \\
2\left[\mathrm{H}_{2}^{\mathrm{HO}_{2}} \mathrm{~B}\right] & \longrightarrow\left[\mathrm{H}_{\mathrm{HO}}^{\mathrm{B}}-\mathrm{B}_{\mathrm{OH}}^{\mathrm{H}_{2}}\right]+\mathrm{H}_{3}
\end{aligned}
$$

Entsprechende Hydroxosalze sind vom Fluorid und von den Alkylen bekannt: $\left[\mathrm{B}_{\mathrm{O}}^{\mathrm{F}_{3}}\right] \mathrm{K}^{13}$, $\left[\mathrm{B}_{\mathrm{OH}^{(} \mathrm{CH}_{3}}^{\left(\mathrm{CH}_{3}\right)} \mathrm{K}^{14}\right.$. Die dehydrierende Polymerisation, welche unter $\mathrm{Z}$ wischenbildung von Radikalen vor sich geht, findet sich auch bei den niederen Boranen. Sie ist ein Ausdruck für die sehr geringe Polarität der B-H-Bindung. (Die Borane sind ja auch keine salzartigen Hydride wie Lithium- oder Calciumhydrid.)

A. Stock und E. Wiberg ${ }^{15}$ vertreten die Ansicht, daß dem Diboran der Charakter einer zweiwertigen Säure zukommt. Die angeführten experimentellen Belege sind aber nicht zwingend. Für die Einwirkung von Basen auf Di- und Tetraboran formuliert E. W i b e r $\mathrm{g}^{16}$ die folgenden Primärreaktionen:

$\left[\mathrm{B}_{2} \mathrm{H}_{4} \mathrm{H}_{2}+2 \mathrm{KOH} \longrightarrow\left[\mathrm{B}_{2} \mathrm{H}_{4}\right] \mathrm{K}_{2}+2 \mathrm{H}_{2} \mathrm{O}\right.$ $\left.\left[\mathrm{B}_{4} \mathrm{H}_{6}\right] \mathrm{H}_{4}\right]+2 \mathrm{Ba}(\mathrm{OH})_{2} \longrightarrow\left[\mathrm{B}_{4} \mathrm{H}_{6}\right] \mathrm{Ba}_{2}+4 \mathrm{H}_{2} \mathrm{O}$

11 Vergl. z. B. S. Sudgen, J. chem. Soc. [London] 1932,1492 .

${ }_{12}$ H. C. B rown, H. I. Schlesinger u. A. B. Burg, J. Amer. chem. Soc. 61, 673 [1939].

${ }_{13} \mathrm{H}$. M e erwein u. W. Pannwitz, J. prakt. Chem. [2] 141, 141 [1934].

${ }_{14}$ E. Krause u. A.v. Grosse, Die Chemie der metall-organischen Verbindungen, Berlin 1937, S. 199.

${ }_{15}$ A. Stock, E. Wiberg, H. Martini u. A. Nickl as, Z. physik. Chem., B odenstein-Festband, 93 [1931].

${ }^{16}$ E. W i b e r g, Ber. dtsch. chem. Ges. 69, 2825 [1936]. 
Die Reaktion (1) ist praktisch überhaupt nicht realisierbar (infolge der Wasserempfindlichkeit des Diborans), auf (2) wird lediglich auf Grund des Basenverbrauchs geschlossen. Wenn bei der Einwirkung von Lauge auf Tetraboran anfänglich kaum oder nur wenig Wasserstoff entwickelt wird ${ }^{17}$, so ist dies noch kein Beweis für das Vorliegen aciden Wasserstoffes, besonders deshalb nicht, weil beim Bor die Fähigkeit zur Bildung von Hydroxosalzen sehr ausgesprochen ist. (Die Formulierung der Basenreaktion des Tetraborans folgt später.) Verbindungen der Art $\mathrm{B}_{2} \mathrm{H}_{4} \mathrm{~K}_{2}, \mathrm{~B}_{4} \mathrm{H}_{6} \mathrm{Ba}_{2}$ ließen sich auch in keinem Falle isolieren. Ihre Darstellung hätte auf dem Wege der Einwirkung von Alkalimetall auf die - lediglich auf Grund ihrer Leitfähigkeit in flüssigem Ammoniak - als Diammoniumsalz $\left[\mathrm{B}_{2} \mathrm{H}_{4}\right]\left(\mathrm{NH}_{4}\right)_{2}$ formulierte Additionsverbindung von Ammoniak an Dibøran gelingen müssen. Tatsächlich wird bei der Ausführung dieses Versuches (in fl. Ammoniak) nur $1 / 2 \mathrm{Mol} . \mathrm{H}_{2}$ auf $1 \mathrm{Mol}$. Diboran in Freiheit gesetzt und die Verbindung $\mathrm{B}_{2} \mathrm{NH}_{8} \mathrm{Na}$ gebil$\operatorname{det}^{18}$. A.B.Burg ${ }^{18}$ schließt hieraus auf den Reaktionsverlauf

$$
\begin{gathered}
\mathrm{BH}_{3}+\mathrm{NH}_{3} \longrightarrow \mathrm{BH}_{3} \mathrm{NH}_{3} \stackrel{\mathrm{NH}_{3}}{\rightarrow}\left[\mathrm{BH}_{3} \mathrm{NH}_{2}\right] \mathrm{NH}_{4} \stackrel{\mathrm{BH}_{3}}{\rightarrow} \rightarrow \\
\stackrel{\longrightarrow}{\longrightarrow}\left[\mathrm{BH}_{3} \mathrm{NH}_{2} \mathrm{BH}_{3}\right] \mathrm{NH}_{4} \frac{+\mathrm{Na}_{2}}{-\mathrm{H}_{2}} \rightarrow\left[\mathrm{BH}_{3} \mathrm{NH}_{2} \mathrm{BH}_{3}\right] \mathrm{Na} .
\end{gathered}
$$

Das Diammoniakat des Diborans ist demnach das Monoammoniumsalz eines propanartigen Anions.

Obwohl sich also fast alle Reaktionen des einfachsten Borhydrides mit der Formel $\mathrm{BH}_{3}$ wiedergeben lassen, ist die Frage nach der Ursache der Dimerisierung des Borins doch nicht zu übersehen; denn es steht die Einheitlichkeit, Formel und Molekulargröße des Diborans fest und es existieren als einheitliche Stoffe charakterisierte Derivate $\mathrm{B}_{2} \mathrm{H}_{5} \mathrm{X}^{19}$. Die Tatsache, daß sich nur vier Wasserstoffatome des Diborans ohne Sprengung des Komplexes alkyl-substituieren lassen - $\left[\mathrm{BH}\left(\mathrm{CH}_{3}\right)_{2}\right]_{2}$ -, leitet zu der Annahme, daß die Bindung der beiden Borinhälften durch zwei Wasserstoffatome, bzw. genauer gesagt durch deren Bindungselektronen $=$ „Bindungen“ bewerkstelligt wird. Da die B-H-Bindung nur ebenso schwach polar ist wie die C-H-Bindung, kommt die Wirkung von Polarisationskräften, wie sie $\mathrm{zu}$ den bekannten „Wasserstoffbrücken"-Mechanismen der $\mathrm{OH}$ - und NH-Bindung führen, kaum in Frage. Das Nächstliegende ist es deshalb, die Wirksamkeit quantenmechanischer "Austauschkräfte" zwischen zwei $\mathrm{B}-\mathrm{H}-\mathrm{Bindungen}$ der beiden Boringruppen anzu-

17 A. St ock u. E. K u ß, Ber. dtsch. chem. Ges. 47, 810 [1914].

18 H. I. S chl es inger u. A. B. B u r g, J. Amer. chem. Soc. 60, 290 [1938]. nehmen, welche das Diboranmolekül aufbauen. Die beiden Boratome des Diborans würden dadurch - indirekt - durch zwei brückenartige Wasserstoffatome verbunden. Dieser Bindungszustand würde der Überlagerung der beiden „Valenzzustände“ (bzw. der Spin-Invarianten des 4-Elektronensystems der beteiligten $\mathrm{B}-\mathrm{H}-\mathrm{Bindungen}$ )<smiles>[BH][BH]</smiles>

entsprechen.

Noch einfacher ließe sich die Diboranformel folgendermaßen wiedergeben<smiles>BCB</smiles>

wodurch auch den sterischen Verhältnissen (Doppeltetraeder) dadurch Rechnung getragen ist, daß die beiden Brücken-Wasserstoffatome übereinanderliegend angenommen sind.

Die wellenmechanische Wechselwirkung zweier Bindungen, welche im Falle des Diborans zu einem äußerst stabilen Anlagerungskomplex führt - und das ist das Neuartige an diesem Hydrid spielt im übrigen ganz allgemein bei der Bildung der - instabilen - Aktivierungskonfigurationen eine Rolle, bei welchen zwei Bindungen an einer Reaktion beteiligt sind:

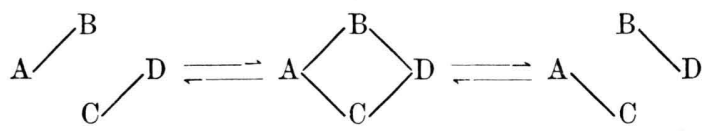

Daß beim dreibindigen Bor die Bildung solcher Komplexe ganz allgemein äußerst leicht erfolgen muß, zeigt sich darin, daß seine Verbindungen mit großer Leichtigkeit Substituenten austauschen bzw. Disproportionierungen erleiden. U. a. beruht hierauf auch die Darstellung der Methylderivate des Diborans ${ }^{20}$ mittels Bortrimethyls:

$$
\left(\mathrm{BH}_{3}\right)_{2}+\mathrm{B}\left(\mathrm{CH}_{3}\right)_{3} \longrightarrow \underbrace{\mathrm{BH}_{3}+\mathrm{BH}_{2} \mathrm{CH}_{3}+\mathrm{BH}\left(\mathrm{CH}_{3}\right)_{2}}_{\mathrm{B}_{2} \mathrm{H}_{6-\mathrm{n}}\left(\begin{array}{l}
\downarrow \\
\begin{array}{l}
\text { kombinierte } \\
\text { A ssoziation }
\end{array}
\end{array} \mathrm{CH}_{3}\right)_{\mathrm{n}}(\mathrm{n}=0-4)}
$$

19 A. St o c k, Ber. dtsch. chem. Ges. 54 A, 156 [1921].

${ }^{20}$ H. I. Schlesing er u. A. O. Walker, J. Amer. chem. Soc. 57, 621 [1935] ; H. I. S ch l e s in ge r, L. H o r vitz u. A. B. B u r g, J. Amer. chem. Soc. 58, 407 [1936]. 
Hierdurch ist eine Brücke zwischen dem reaktiven Verhalten der übrigen Verbindungen des Bors und dem Assoziierungsmechanismus des Borins geschlagen.

Im Rahmen einer vollständigen Diskussion der Konstitution des Diborans wären endlich auch die Ergebnisse physikalischer Untersuchungsmethoden heranzuziehen. Diese haben sich bis jetzt auf die Messung der Verbrennungs- und spezifischen Wärme, der Röntgen- und Elektronen-Interferenzen, die massenspektrometrische Analyse, das Utraviolett- und Ultrarotspektrum, den R amanEffekt und die Bestimmung des elektrischen und magnetischen Moments erstreckt. Bei einer kritischen Beurteilung dieser Ergebnisse ergibt sich, daß sie in allen solchen Fällen, wo eine klare Entscheidung möglich ist, für das entwickelte Brückenmodell des Diborans sprechen ${ }^{21}$.

Die vorgetragene Auffassung der Borinassoziation führt nun aber auch zu einer Erklärung der Bildungsursachen der höheren Borwasserstoffverbindungen oder „Polyborane“. Bei näherer Betrachtung findet man, daß jeder der bis jetzt isolierten flüchtigen Borwasserstoffe sich aufgebaut denken läßt aus einfacheren, der normalen Dreiwertigkeit (bzw. besser Dreibindigkeit) des Bors entsprechenden Hydriden $\mathrm{B}_{n} \mathrm{H}_{n+2}$ (vergl. die Paraffine $\mathrm{C}_{\mathrm{n}} \mathrm{H}_{2 \mathrm{n}+2}$ ):

$\mathrm{B}_{4} \mathrm{H}_{10}=\mathrm{B}_{2} \mathrm{H}_{4}+2 \mathrm{BH}_{3}$

$\mathrm{B}_{5}^{4} \mathrm{H}_{11}^{10}=\mathrm{B}_{3}^{2} \mathrm{H}_{5}^{4}+2 \mathrm{BH}_{3}$

$\mathrm{B}_{6} \mathrm{H}_{12}=\mathrm{B}_{4} \mathrm{H}_{6}+2 \mathrm{BH}_{3}^{3}$

$\mathrm{B}_{5} \mathrm{H}_{9}=\mathrm{B}_{2} \mathrm{H}_{4}+\mathrm{B}_{3} \mathrm{H}_{5}$

$\mathrm{B}_{6} \mathrm{H}_{10}=2 \mathrm{~B}_{3} \mathrm{H}_{5}$

$\mathrm{B}_{10} \mathrm{H}_{14}=2 \mathrm{~B}_{3} \mathrm{H}_{7}$

Die angeführten Komponenten $\mathrm{B}_{\mathrm{n}} \mathrm{H}_{\mathrm{n}+2}$, ,Polyborine", sind dabei - infolge ihres Assoziierungsbestrebens - für sich als ebenso unbeständig anzunehmen wie das Borin selbst. (Als Derivate des Diborins können $\mathrm{B}_{2} \mathrm{Cl}_{4}{ }^{22}, \mathrm{~B}_{2}(\mathrm{OH})_{4}$ und $\mathrm{B}_{2}(\mathrm{OR})_{4}{ }^{23}$ betrachtet werden; die Hypoborate, zum Beispiel $\left[\mathrm{B}_{2} \mathrm{H}_{4}(\mathrm{OH})_{2}\right] \mathrm{K}_{2}$, sind seine Hydroxosalze.)

Die erste wasserstoffreichere Reihe der Polyborane kann man aus je einem Di-, Tri-, Tetraborin-Molekül und zwei Boringruppen bilden, welche die assoziationsfähigen endständigen $-\mathrm{BH}_{2}-$ Gruppen des Polyborins mittels des Borinbindungssmechanismus absättigen. Es führt dies zu offenen Ketten (I, IV) mit der verdreifachten allgemeinen Polyborinformel $\mathrm{B}_{\mathrm{x}} \mathrm{H}_{\mathrm{x}+6}$ :

21 Im einzelnen muß auf die eingangs zitierte aus führliche Arbeit d. Verf. verwiesen werden.

${ }_{22}$ A. St ock, A. B r and t u. H. F is cher, Ber. dtsch. chem. Ges. 58, 643 [1925].

23 E. W i ber g u. W. R u s c h m an n, Ber. dtsch. chem. Ges, 70, 1393 [1937].

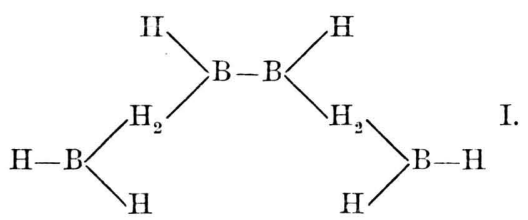

Im Falle des Tetraborans konnte die Struktur (I) durch die Wurtz sche Synthese $2 \mathrm{~B}_{2} \mathrm{H}_{5} \mathrm{~J}+2 \mathrm{Na}$ $\rightarrow \mathrm{B}_{4} \mathrm{H}_{10}+2 \mathrm{NaJ}$ experimentell bewiesen werden ${ }^{24}$. Der Selbstzerfall des Tetraborans ${ }^{25}$ in Diboran und Borwasserstoffe höheren Molekulargewichts steht in ausgezeichnetem Einklang mit dieser Formulierung, da es hiernach zwei Borinradikale enthält:

$$
\begin{aligned}
& \mathrm{B}_{4} \mathrm{H}_{10} \longrightarrow 2 \mathrm{BH}_{3}+\mathrm{B}_{2} \mathrm{H}_{4} \\
& \downarrow \cdots \cdots \rightarrow \downarrow \text { Dehydrierende Polymerisation } \\
& \left(\mathrm{BH}_{3}\right)_{2} \text { höhermolekulare Borwasserstoffe }
\end{aligned}
$$

Auch andere Umsetzungen des Tetraborans können als Reaktionen seiner Komponenten gedeutet werden, z. B. die Einwirkung von Laugen, welche zu Hypoboraten führt ${ }^{20}$ :

$$
\begin{aligned}
& \mathrm{B}_{4} \mathrm{H}_{10} \longrightarrow 2 \mathrm{BH}_{3}+\mathrm{B}_{2} \mathrm{H}_{4} \\
& \downarrow+2 \mathrm{OH}^{-} \quad \downarrow+2 \mathrm{OH}^{-}
\end{aligned}
$$

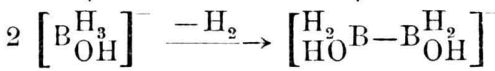

Während aus der Komponente Diborin unmittelbar ihr Hydroxosalz (Hypoborat) gebildet wird, geht die Komponente Borin über deren Hydroxosalz (wie bereits beim Diboran ausgeführt wurde) erst durch Dehydrierung in Hypoborat über. Infolge der größeren Stabilität des Tetraborans gegenüber Wasser hebt sich hier auch der erste Schritt der Hypoboratbildung aus Borin deutlich ab: es ergab sich nämlich, daß bei der Einwirkung von Laugen auf Tetraboran zunächst kein Wasserstoff entbunden wird.

Die zweite, wasserstoffärmere Reihe von Polyboranen mit der verdoppelten Borinformel $\mathrm{B}_{\mathrm{y}} \mathrm{H}_{\mathrm{y}+4}$ setzt sich nur aus Polyborinen zusammen. Da diese die Funktion der Assoziationsfähigkeit an mehreren Stellen im Molekül besitzen, kann die Anlagerung zu Doppelketten bzw. cyclischen Konfigurationen führen. Es ergeben sich damit für diese Reihe (das Diboran selbst kann an den Anfang gestellt werden) die Strukturbilder II, III, V.

$\approx 4$ A. Stock u. E. Pohland, Ber. dtsch. chem. Ges. 59, 2223 [1926].

$\because 5$ A. Stock, K. Friederici u. O. Priess, Ber. 46, 1959, 3353 [1913].

${ }_{20}$ A. Stock u. E. Kuß, Ber. dtsch. chem. Ges. 47, 810, [1914]. 
<smiles>[1H][PH2+]</smiles><smiles>[B]C[B][B][BH]</smiles>

II.<smiles>[10BH2]</smiles>

$\mathrm{B}_{6} \mathrm{H}_{10}$<smiles>[B][B][B][B][B][B][B]</smiles>

III.

$\mathrm{B}_{5} \mathrm{H}_{11}$ und $\mathrm{B}_{5} \mathrm{H}_{9}$ stehen zueinander im Verhältnis von Pentan und Cyclopentan (IV-V); daß $\mathrm{B}_{5} \mathrm{H}_{11}$ mit Ammoniak unter Dehydrierung dasselbe Ammoniak $\mathrm{B}_{5} \mathrm{H}_{9}\left(\mathrm{NH}_{3}\right)_{4}$ liefert wie $\mathrm{B}_{5} \mathrm{H}_{9}$, wird hierdurch verständlich ${ }^{27}$. Ebenso steht mit der dargelegten Formulierung in Einklang, daß $\mathrm{B}_{10} \mathrm{H}_{14}$ aus $\mathrm{B}_{5} \mathrm{H}_{11}$ durch Abgabe von vier Molekülen Wasserstoff entsteht (IV-VI-III) ${ }^{28}$.
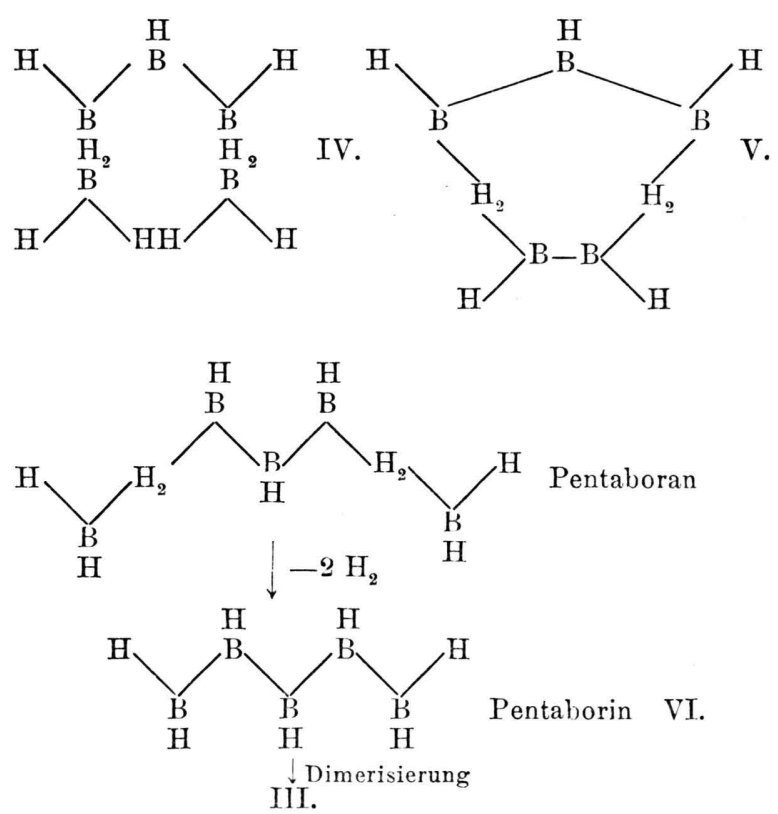

Die bereits aus den Formelbildern klar ersichtliche Differenzierung der beiden Gruppen von $\mathrm{Hy}$ driden tritt auch in ihren Eigenschaften deutlich zutage. Die erste Reihe schmilzt niedriger $\left(\mathrm{B}_{4} \mathrm{H}_{10}\right.$ $\left.-120^{\circ}, \mathrm{B}_{5} \mathrm{H}_{11}-128^{\circ}, \mathrm{B}_{6} \mathrm{H}_{12}-90^{\circ}\right)$ als die zweite $\left(\mathrm{B}_{5} \mathrm{H}_{9}-47^{0}, \mathrm{~B}_{6} \mathrm{H}_{10}-65^{0}, \mathrm{~B}_{10} \mathrm{H}_{14}+97^{0}\right)^{27}$. Jene ist unbeständiger als diese $-\mathrm{B}_{4} \mathrm{H}_{10}, \mathrm{~B}_{5} \mathrm{H}_{11}, \mathrm{~B}_{6} \mathrm{H}_{12}$ höchst zersetzlich, was offenbar in dem Gehalt an Borinradikalen begründet ist $-\mathrm{B}_{5} \mathrm{H}_{9}$ und $\mathrm{B}_{6} \mathrm{H}_{12}$ verhältnismäßig beständig ${ }^{27}, \mathrm{~B}_{10} \mathrm{H}_{14}$ am beständigsten $^{29}$. Wasser hydrolysiert die wasserstoffreicheren Hydride $\mathrm{B}_{2} \mathrm{H}_{6}$ augenblicklich, $\mathrm{B}_{4} \mathrm{H}_{10}$ ziemlich rasch $-\mathrm{B}_{5} \mathrm{H}_{9}, \mathrm{~B}_{6} \mathrm{H}_{10}$ und $\mathrm{B}_{6} \mathrm{H}_{14}$ dagegen nur langsam ${ }^{29}$.

Da keine Borwasserstoffe mit freien $-\mathrm{BH}_{2}$-Gruppen existieren können, müssen die sämtlichen realen Polyborane den beiden Formeln $\mathrm{B}_{\mathrm{x}} \mathrm{H}_{\mathrm{x}+6}(\mathrm{x} \geq 4)$ und $\mathrm{B}_{\mathrm{y}} \mathrm{H}_{\mathrm{y}+4}(\mathrm{y}>4)$ genügen, wodurch die Nichtexistenz anderer Formeltypen erklärt wird. In der zweiten Reihe fallen die Glieder mit $\mathrm{y}=4$ und $y=8$ wohl deshalb aus, weil auf diese Weise keine spannungsfreien Ringsysteme gebildet werden können.

Außer den bisher betrachteten, mehr oder minder flüchtigen Borwasserstoffen sind noch nichtflüchtige Hochpolymere bekannt ${ }^{30}$. Es wird diese Tatsache nicht überraschen, da die Polyborinassoziation nicht nur zu in sich geschlossenen Ringen zu führen braucht, sondern auch fortlaufende und verzweigte Ketten entstehen können, wie sie aus der Chemie der Hochpolymeren bekannt sind.

Die merkwürdige Zusammensetzung der höheren Borwasserstoffverbindungen ist also die einzigartige Folge der Überschneidung zweier Bindungsmechanismen, deren einer in der für die Borchemie charakteristischen Kopplung von Wasserstoffbindungen besteht, während der andere, die direkte Bor-Bor-Atombindung, bereits die verwandtschaftlichen Beziehungen zur Chemie der Kohlenstoffund Silicium-Verbindungen erkennen läßt.

27 A. Stock u. E. Pohland, Ber. dtsch. chem. Ges. 59, 2210 [1926].

${ }_{28}$ A. Stock u. W. Siecke, Ber. dtsch. chem. Ges. 57, 565 [1924].

29 A. St ock, Ber. dtsch. chem. Ges. 54 A, 155 [1921].

30 Vergl. z. B. A. Stock, K. Friederici u. O. Priess, Ber. dtsch. chem. Ges. 46, 3361 [1913]. 